\title{
Unified Power Flow Controller (UPFC) Modeling and Analysis Technique by using Matlab and EMTP
}

\author{
Tariq Masood ${ }^{1}$, Nasir Jameel ${ }^{2}$, Suhail Aftab Qureshi ${ }^{3}$, Muhammad Tajammal ${ }^{4}$ \\ Samer Karim Haider ${ }^{5}$, Ghulam Hashmi ${ }^{6}$ \\ ${ }^{1}$ Asset Integrity Department, Dukhan Operations, Qatar Petroleum, Email: T.Masood.Dr@ieee.org \\ ${ }^{2}$ Maintenance Department Schlumberger Surface Maintenance Australia: Email:nasirjameel130@gmail.com \\ ${ }^{3}$ University of Engineering and Technology, Lahore Pakistan: Email: tiger suhail@hotmail.com \\ ${ }^{4}$ University of Hail, Hail, Saudi Arabia: Email: mt.chughtai@uoh.edu.sa \\ ${ }^{5}$ Maintenance Department QAFAC Doha, Qatar: Email: samerk@qafac.com.qa \\ ${ }^{6}$ Power System Engineering Department Saudi Aramco, Dhahran, Saudi Arabia: Ghulam.hashmi@,Aramco.com
}

\begin{abstract}
The UPFC is a state-of-the-art control device that has the ability to enhance electrical power system network operation more precisely and effectively. This device encompasses of shunt and series parts to readjust capacitive and inductive compensation of the network based on their ratings to dynamically control the operating parameters of the transmission-network. These dynamic parameters comprise of phase angles, line impedance and line voltage. During the detailed investigation, a set of equations are introduced to compute UPFC operations/control behaviour on shared two bus system networks. In fact, the EMTP and Matlab models are introduced and customized to determine and validate UPFC effectiveness with precision. In this investigation, a pragmatic approach demonstrated and provided roadmap for the planning and control electrical engineer to evaluate UPFC operational behaviour and its significant impact on Electrical power transmission network operations. This device has the ability to address all types of time based contingencies in order for a seamless operation.
\end{abstract}

Key words: EMTP, FACTS, Matlab, UPFC, TNA, Reactive Power

\section{Introduction}

Effectively speaking, the electrical power system operations/control have never been ideal. Technically, when the industry and domestic power consumption demand increases, the electrical power utilities will load the equipment at maximum capacity in line with their operating and design range. Therefore, the load fluctuates as per need and the power utilities equipment oscillate and reach to its maximum operating range to meet the required power demand and vary the operating envelop of the Power system network. Therefore, the GTO based UPFC device is a solution to control and address the time-based power system contingencies. The novelty of GTO Thyristors has the ability to provide robust and flexible control to the power transmission network without any operational constraint. In this investigation, a UPFC model developed and simulated by integrating following operating parameters; the series part of the UPFC contains the operating parameters as given (1) line voltage $400 \mathrm{kV}$ (2) $400 \mathrm{Mvar}$ Thyrister Control Series Capacitor (TCSC) and shunt part of the UPFC contains operative parameters as given (1) line voltage $220 \mathrm{kV}$ (2) 200Mvar static synchronous compensator (STATCOM) on the GCC power grid. Both parts of the UPFC provides robust control to stabilize line voltage, phase-angle and line impedance. The model outcomes reveal that the UPFC augments power system operations and control on the GCC power grid by large. The Matlab model corroborated to be a very strong methodical tool for emergent and examining bespoke model's process operations characteristics. Control competence of the UPFC device to meet essential growing electrical power system demand and also to sustain uniform operations on the GCC Power grid. The Matlab codes developed and analyzed their performance with its validation process and positive impact. Following key parameters have been discussed as listed below. Section (I) address the paper abstract and introduction, section (II) presents the Matlab simulation and integrations codes. Section (III) demonstrates the series and shunt part of a UPFC device on the electrical power network. Section (IV) contributes operational assessment and substantiation of a UPFC device's positive impact on the Power operations network. Section (V) substantiate the simulation model by using Matlab, Transient Network Analyzer (TNA) and Electromagnetic Transient program(EMTP).

\section{Mathematical Model of UPFC Device}

In this section, numerous mathematical equations are developed and synthesized on two bus electrical power system operations. This device's characteristics are established in line with d-q transformation, and very much compatible with small, large and multifaceted electrical power network operations.

A. $\quad d-q$ Transformation and Equations Configuration In this investigation, the voltage-source-branch comprised of series with R-L operative parameters which present transformer and power line transmission. However, d-q presents the direct and quadrature projection of the vectors.

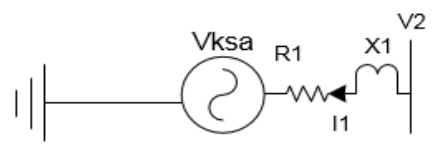

Figure 1. d-q analysis one-line diagram constituted B. Power Network Performance Equations

Figure 1 designates the UPFC device is employed on the two-bus system and its meticulous computation. This section describes the set of tailored equations which are an 
essential to develop the model and synthesize in two bus system and simulate to achieve desired results on the electrical power system.

$$
\begin{aligned}
& R_{1}, X_{1}=L_{1} \omega, R_{s} \\
& X_{s}=L_{s} \omega
\end{aligned}
$$

Equation (1) and (2) values are computed by considering short circuit power references. There are two critical parameters must be considered (1) $\mathrm{XR}=\mathrm{X} / \mathrm{R}$ ratio (2) power system short-circuit to witness required results. Further assessment can be performed as given below. Wherein the $\mathrm{X}=\mathrm{L} \omega$ and the operative parameters of the electrical power transmission-network are computed rigorously to integrate, by using the equations (3) and (4) as given below:-

$$
\begin{aligned}
& R=\frac{V_{n}^{2}}{S_{s c} \sqrt{1+X R^{2}}} \\
& L \omega=X R
\end{aligned}
$$

Wherein the $\mathrm{V}_{\text {-ksa }}$ and $\mathrm{V}_{\text {-kt }}$ are computed vector voltages. In order to confirm and authenticate that the infinite voltage source is computed to deliver the required P-2 and Q-2 on the power network. When an electrical power system's technical losses are not considered then an unconventional robust approach can be espoused-In general, Equation (5) is used to compute and measure $\mathrm{V}_{1}$ value without UPFC device in between the Kingdom of Saudi Arabia and Kuwait.

$$
V_{1}=R I+j L \omega I+V_{2}
$$

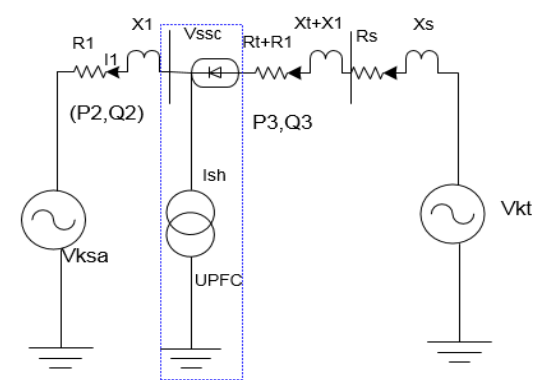

Figure. 2. UPFC device on the KSA and Kuwait

\section{Power network Main Algorithm}

In the main algorithm, numerous distinctive control models are developed and demonstrated their results as discussed in Figure 3. (I) In the first section of the algorithm model which indicates how to manage the power of the short circuits: $\mathrm{S}_{\mathrm{SRC} 1}$ and $\mathrm{S}_{\mathrm{SRC} 2}$. (II) In the second section of the algorithm model how to control the reactive power current injection from the shunt-part inverter $i_{\text {shq }}$ of the UPFC. (III) In the third section of the algorithm model how to control the series voltage amplitude Vs and phase-shift PHIs. In this investigation, a MATLAB model is devised and tailored to align with the power system operations at various operating conditions on the GCC network. This is highly conceivable to constitute and ascertain the control loop by incorporating several controls and operating parameters which provide a roadmap to determine how much power is flowing in the power transmission system voltage at $\mathrm{V} 2$, and $\mathrm{X} / \mathrm{R}$ ratio.

\section{Convergence Operating and Control Criteria}

For any type of time-based computational to accomplish the conjunction state of the Matlab simulation model, this is an indispensable matter to provide high quality results. Equation (6) indicates the time-based convergence for two bus system power network operating parameters herein discussed, firstly is the extent voltage V2d and secondly is the current in series i3. In the First extent V2d voltage validates the appropriate convergence of the capacitive/inductive mode of shunt part of the UPFC. In the second extent i3 series current validates appropriate convergence for the series voltage injection in the projected system by the UPFC Controller.

$$
\frac{V_{2 d}^{(n+1)}-V_{2 d}^{n}}{V_{2 d}^{n}}<10^{-4}, \frac{i_{3}^{(n+1)}-i_{3}^{n}}{i_{3}^{n}}<10^{-4}
$$

The Matlab model conjunction benchmarks are very significant and efficient for all the functional and control parameters that have been deliberated in the investigation. The Matlab Model designates that the UPFC device is responding within 5 to $45 \mathrm{~ms}$ in line with effective points wherein the electrical power system performs forcefully. Ultimately, accumulative the method and reducing the number of iterations to complete the compensation process. However, this finally produces further enhancement in shunt and series parts of the UPFC respond to compensate capacitive/inductive mode of operations essentially. Figure 3 designates in feature how the UPFC investigation algorithm model accomplishes, which is the core gradient, and is developed in a sequential order.

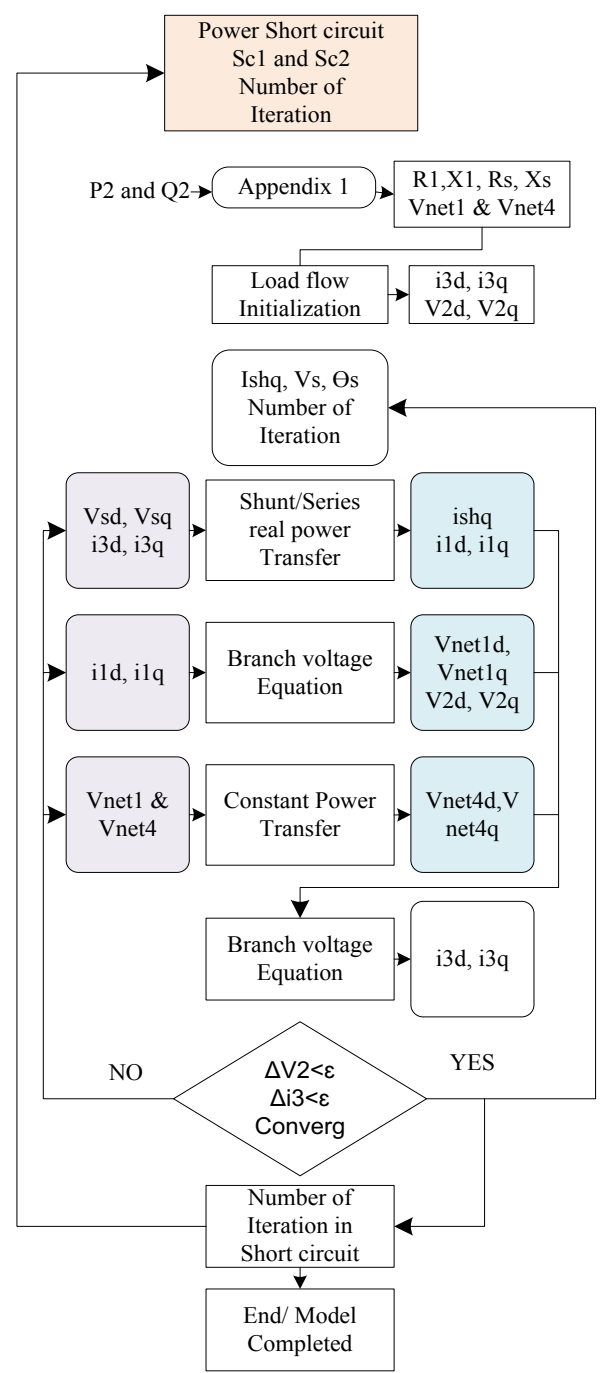

Figure 3 Power System Model Analyses Core Algorithm 


\section{UPFC Model Compensation}

The Matlab model validates the actual compensation influence by using shunt and series parts of the UPFC on the the GCC power grid [4]. Herein, following control and effective control parameters (R1, Rs, X1 and Xs) are computed in line with given short circuit strength at both buses on the GCC power grid. How the $\mathrm{V}_{\text {net1 }}$ and $\mathrm{V}_{\text {net2 }}$ voltages are controlled on the power transmission network

$$
P_{2}^{(0)}=700 M W, Q_{2}^{(0)}=-500 M v a r
$$

$\mathrm{V}_{\mathrm{ksa}}$ voltage is monitored and controlled at substation (KSA) $\mathrm{V}_{2}=400 \mathrm{kV}=1 \mathrm{p} . \mathrm{u}$, and the UPFC shunt part can deliver reactive power up to $+/-250$ Mvar .

\section{A. Impact of Short Circuit at the network}

Investigation indicates that the Matlab model has a stupendous ability to carry-out numerous parametric analyses. Figure 4 denotes the $\mathrm{V}_{\mathrm{ksa}}$ voltage magnitude at $\mathrm{V}_{2}$ to activate and readjust inductive/ capacitive mode to compensate by the shunt part of UPFC device at numerous levels of short-circuit.

\section{$\mathrm{S}_{\mathrm{scl}}=[1500: 1200: 2500: 3000: 3500: 4000: 4500] \mathrm{MVA}$}

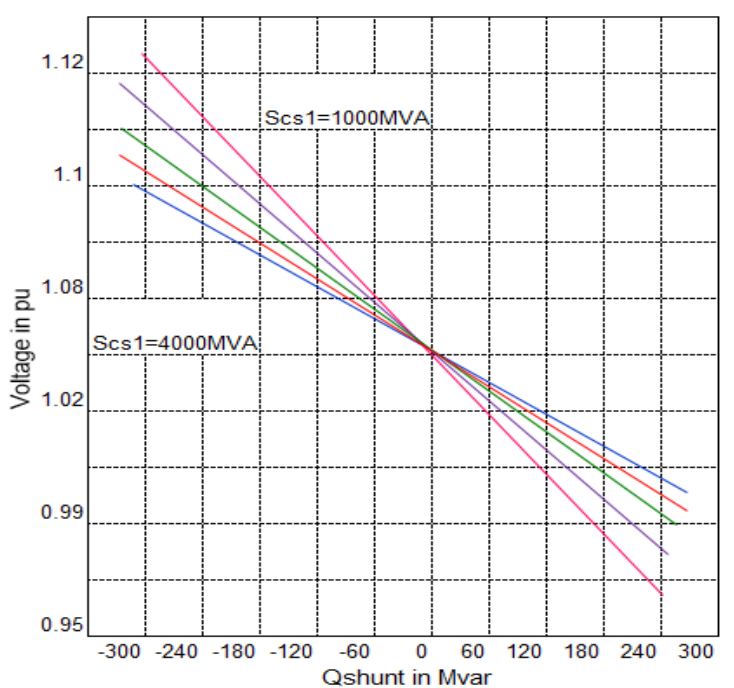

Figure 4 Model voltage level at various Scs1 at V2

Figure 4 also designates that UPFC shunt-part has a ability to address the voltage deviation from $+/-4.5 \%$ to $+/-$ $8.5 \%$ for the thorough numerous levels of short-circuit of power network. Figure 5 (a) and (b) reveal the reactive and real electrical power flows by the UPFC shunt-part accustomed instinctively from capacitive to inductive mode of operations from -350 to +350 MVA.

It also denotes that the active power flow varies from $900 \mathrm{MW}$ to $1000 \mathrm{MW}$, versus reactive power flow which is coordinated by readjusting inductive/capacitive mode of operations up to 500 Mvar. The active power perceived to be boosted in between 120MW and 90MW while the Ssc1 bears the values of 1500MVA or 4500MVA correspondingly [5].

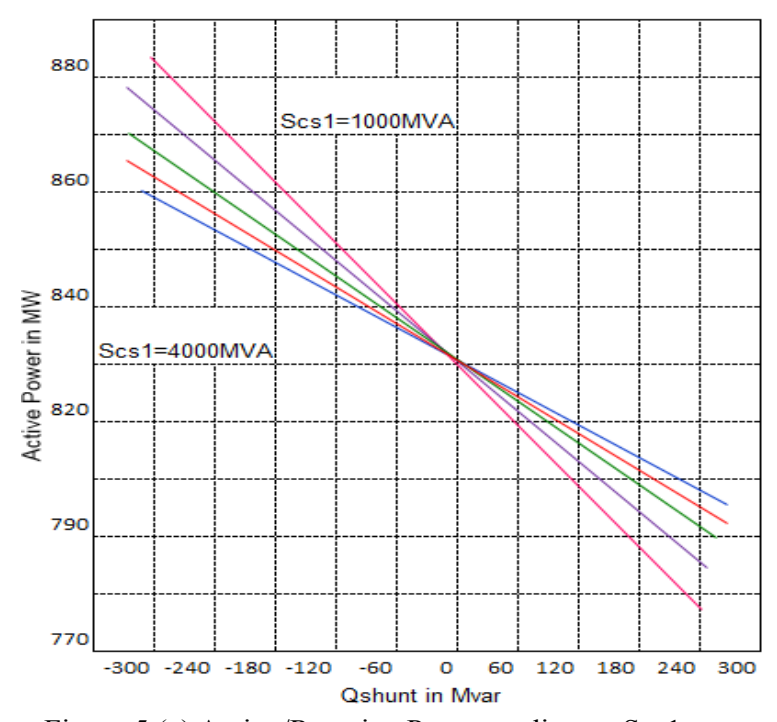

Figure 5 (a) Active/Reactive Power at diverse Scs1 at V2 voltage level

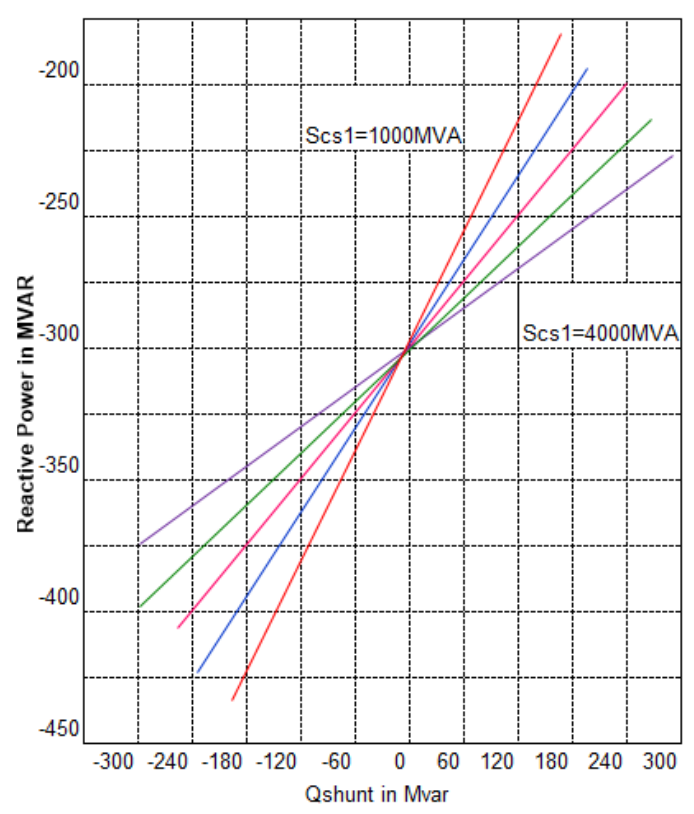

Figure 6 (b) Active/Reactive Power at diverse Scs1 at V2 voltage level

\section{Series Part of UPFC}

A. Series Voltage Injection impact on the Power System

Series part inverter of the UPFC device injects the voltage from the given values $(\mathrm{Vs}) \mathrm{rms}=[$ from $-20.3 \mathrm{kV}$ to $+20.3 \mathrm{kV}]$ and $\varphi=\left[\right.$ from $-90^{\circ}$ to $\left.+90^{\circ}\right]$ leakage drop of the series transformer has been discounted as given in Figure 6 . 


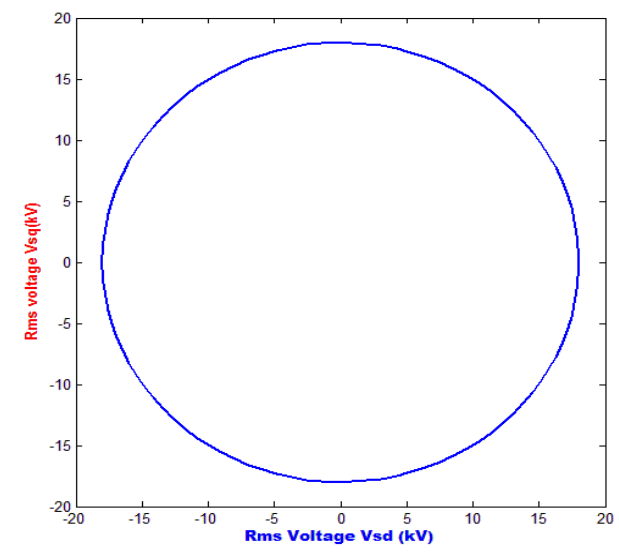

Figure 7 UPFC series voltage injected

B. Reactive Current \& Series Voltage Injection Management

Maximum/minimum reactive electrical power (+/$500 \mathrm{Mvar}$ ) that can be dispensed by the shunt part of the UPFC which has been computed and presented their results in Figures 7 through 9. However, there is no series voltage injection $\mathrm{Vs}=0 \mathrm{kV}$. The series voltage injected on the power system is shown in Figure 6, and resultant real power flow is shown in Figure 7. In Figure 7, the ellipse at the bottom designates that when UPFC shunt-part was on capacitive mode and dispensed -350Mvar and the series voltage Vs distinct subsequently to the diverse segments on the perimeter of the circle as exhibited Figure 6.

Figure 7 expresses the ellipse from the top. This is due to the condition when UPFC shunt-part was on inductive mode and has dispensed $+350 \mathrm{Mvar}$. The injected series voltage Vs miscellaneous suitably to diverse segments on the perimeter of the circle as exhibited in Figure 6

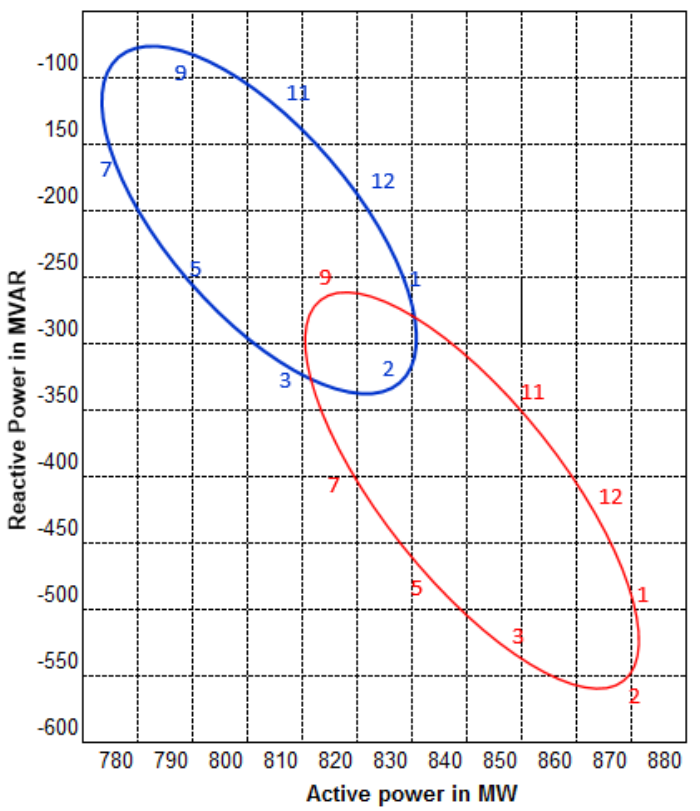

Figure 8 active/reactive power diagram [+/-250 Mvar]

Figure $8 \& 9$ reveal on the GCC power network voltage deviation profile from V2 and V3 as related to numerous operating segments as discussed in Figure 6. For instance, in segment 2 on the top ellipse in Figure 6, as it observed $\mathrm{Vsd}=0$ and $\mathrm{Vreq}=-20.3 \mathrm{kV}$. In fact the UPFC device's shunt- part acquired a reference value which is equal to +500 Mvar. Figure 7 and 8 illustrate the graphical representation of the Matlab model results as given.

P3 $=900 \mathrm{MW}, \mathrm{Q} 3=-350 \mathrm{Mvar}, \mathrm{V} 2=0.985 \mathrm{pu}$ and V3 $1.18 \mathrm{pu}$

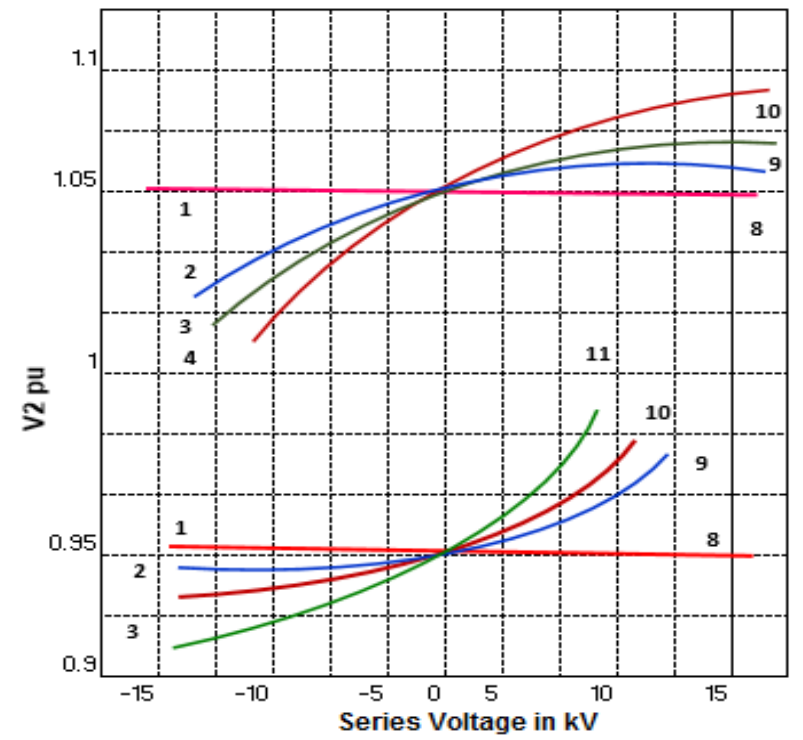

Figure 9 Transmission-network voltage at shunt-part V2 of the UPFC device

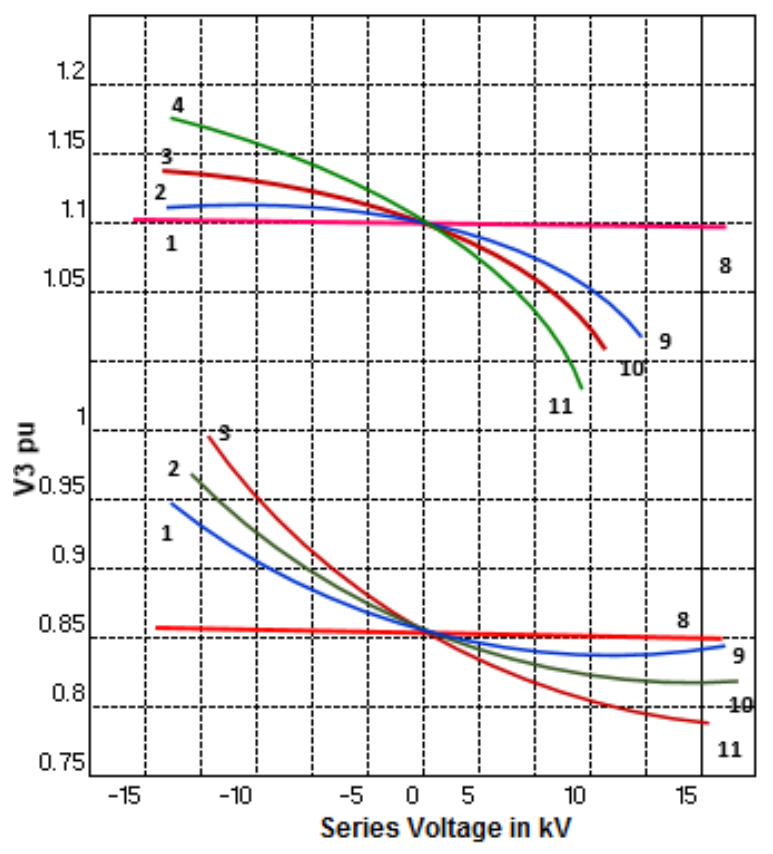

Figure 10 Power Transmission network voltage V3

The main operational encounter is that if electrical transmission line-voltage extended up to $1.18 \mathrm{pu}$, means that the UPFC is operating at its determined operative capability range, which stances as a key concern.

In order to witness its determined benefit from the UPFC device, it is an essential to intensify the insulation of the network to sustain up to $1.18 \mathrm{pu}$, this is main power transmission network major bottleneck. Therefore, the UPFC device operations have to be coordinated precisely to its threshold value at assured operating level of power transmission line-voltage. This avoids an unforeseen overvoltage effective operative scenario, which may lead to calamitous and devastating failure of the power 
transmission network.

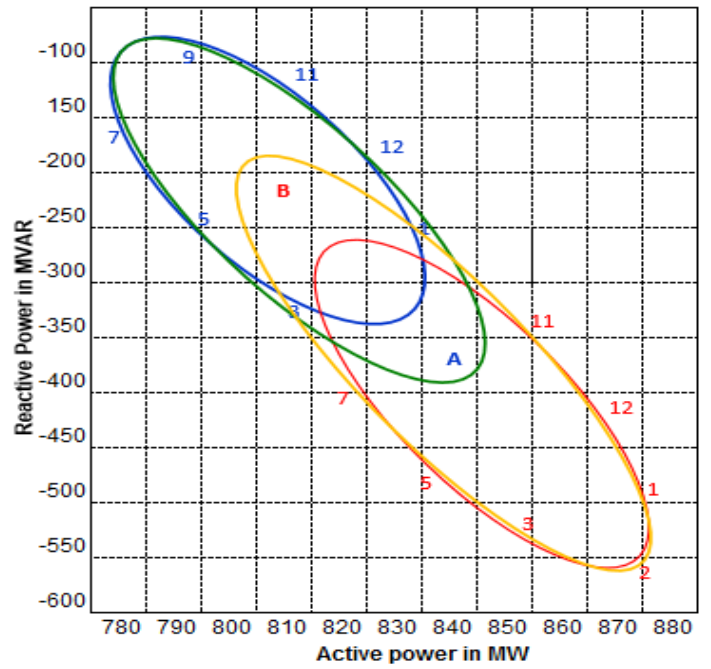

Figure 11 Power diagram for Scs1

[2500MVA;4000MVA]

\section{Positive Impact of the UPFC for Different Power Short Circuit}

Four power ellipse sketches reveal the adaptable power flow region with more precisely. It also designates the two short circuit values of 2500MVA and 4500MVA at two bus system Kingdom of Saudi Arabia.

\section{Validation by Using EMTP}

An EMTP (Electromagnetic transient program) model was developed to tailor the operative parameters in order to substantiate and integrate the Matlab-model's integrity. Therefore, the model authenticates that Matlab-model codes are developed and simulated to witness desired results without any process uncertainty.

\section{E. TNA (Transient network Analyzer) Outcomes}

The UPFC device was unified and constituted at two bus system matching to as revealed in Figure 2 and it was also unveiled promising result on the Transient net-work Analyzer (TNA). This provides roadmap to measure and uphold that the current analyses technique is very much feasible and viable to serve the purposeful objective of the power network. Figure 11 reveals the attained results from the TNA analyzer. The UPFC device's series-part inserts the series voltage Vs $=20.3 \mathrm{kV}$, $\mathrm{Phi}_{\mathrm{s}}=0^{\circ}$ to $360^{\circ}$ periodically at $30^{\circ}$ the UPFC device's shunt-part establishes the reference voltage was attained and extended as required, which was equal to $0.98 \mathrm{pu}$. In Figure 11, the ellipse determines the pretend power flow region of the UPFC device operations on the GCC power net-work.

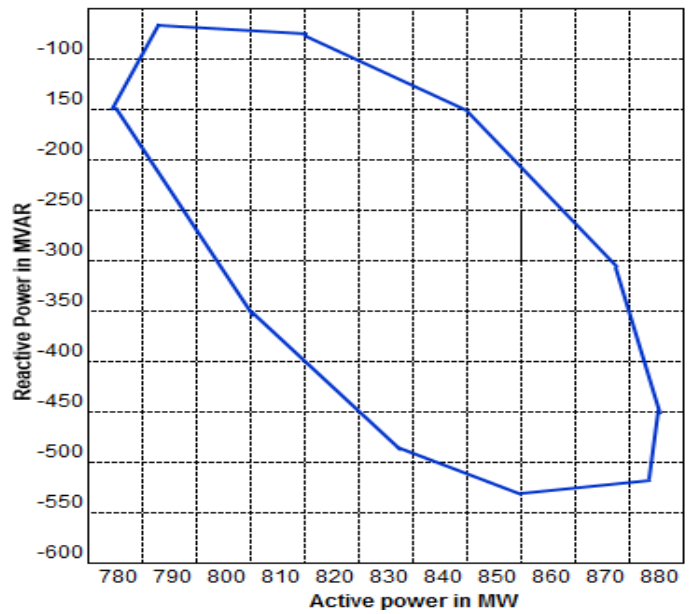

Figure 12 Transient Network Analyzer Power System Operational Trends

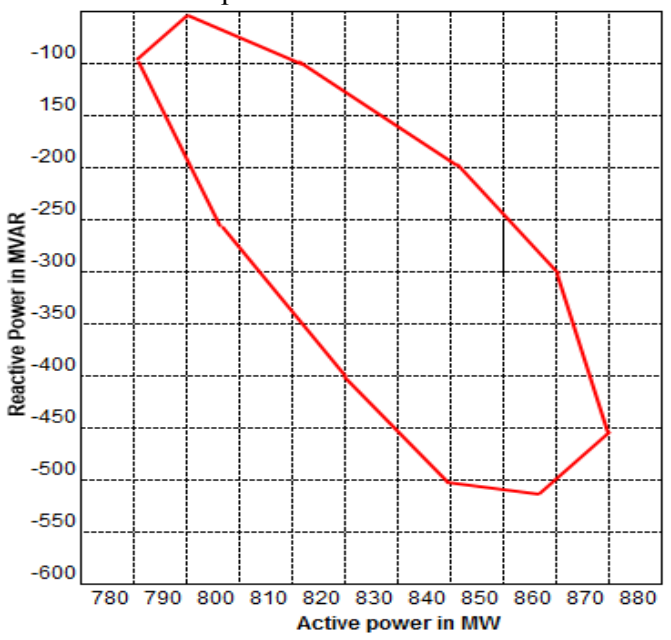

Figure13 Matlab Power system operational trends.

Figure 11 and 12 both determine the attained numerical values from the simulated model. The primary discrepancy was because of the exhaustive TNA model analyzer outcomes, which is replica as express in Figure 2. Herein the transmission line's impedance transfer in between two bus system was also investigated during the simulation. The TNA model was constituted by using proposed and actual UPFC device operative parameters envelope. This reveals marginally different reactive powers consumption than the computation to determine the equipment's constraints. The purpose of this paper is to stipulate a very simple modeling technique to carryout multiple analyses to regulate matching values or estimation.

\section{Conclusion}

This investigation presents the favorable operational impact of the UPFC device on two bus system on the GCC power grid, which is evaluated by using Matlab simulation. The Matlab tool is very much pragmatic to recognize and govern the UPFC device distinctive performance. The UPFC device has an adaptable ability to employed in between Kuwait and the Kingdom of Saudi Arabia. EMTP is used to endorse the Matlab model's integrity and reliability. TNA model's has an inveterate response on the GCC network's operational reliability and integrity of the estimation, and constituted a new power system modeling technique to achieve desired result. The 
Matlab codes are developed step by step to carry-out fast, rational and to achieve logical investigation outcomes to determine UPFC device effective implementation on the GCC power grid.

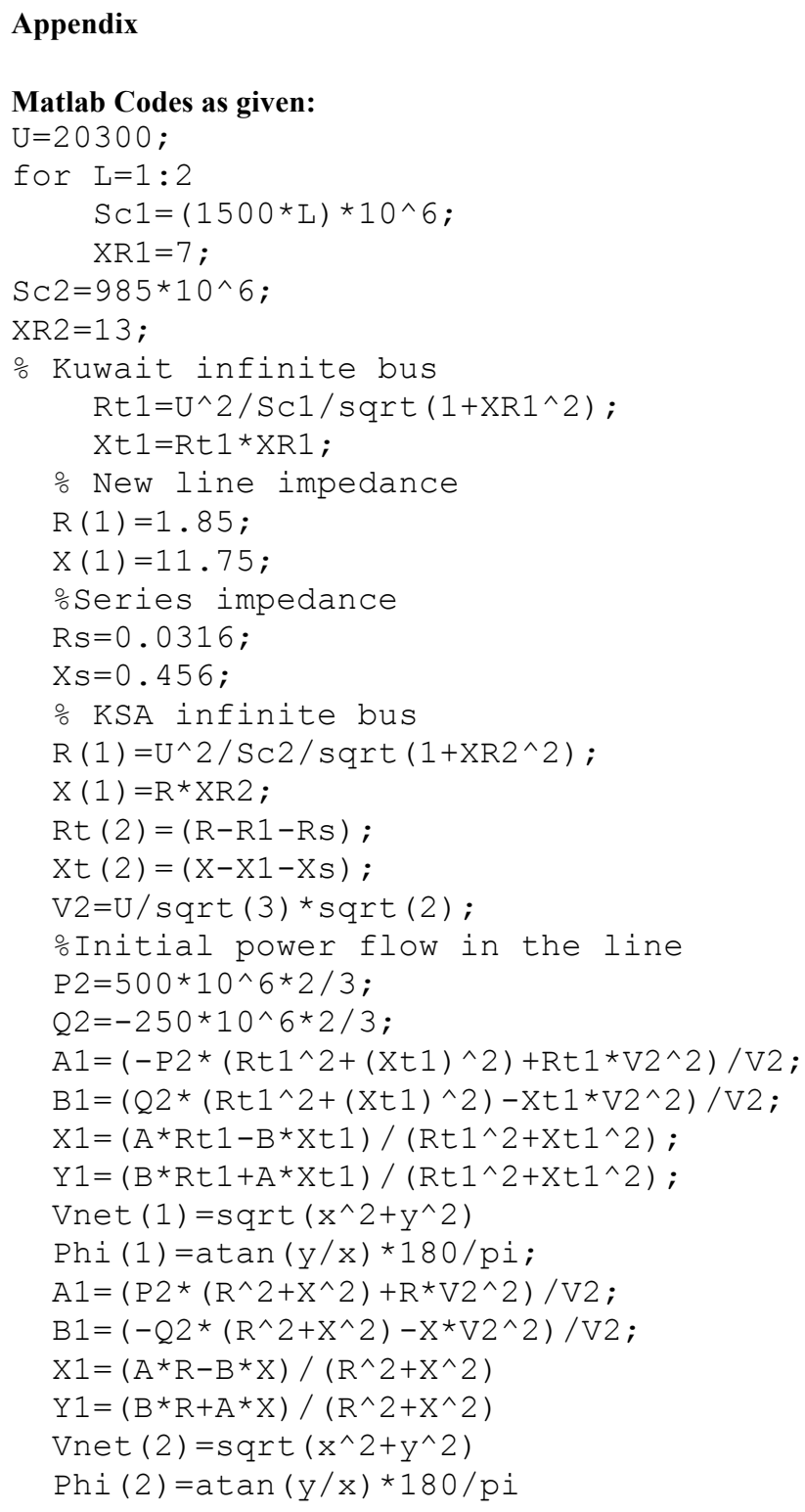

UPFC Mathematical Model:

$$
\begin{aligned}
& \frac{1}{\omega_{s}} \frac{d}{d t} i_{d 1}=\frac{k_{1} d c}{L_{s 1}} \cos \left(\alpha_{1}+\theta_{1}\right)+\frac{\omega}{\omega_{s}} i_{q 1}+\frac{R_{s 1}}{L_{s 1}} i_{d 1}-\frac{V_{1}}{L_{s 1}} \cos \theta_{1}(6) \\
& \frac{1}{\omega_{s}} \frac{d}{d t} i_{d 1}=\frac{k_{1} d c}{L_{s 1}} \sin \left(\alpha_{1}+\theta_{1}\right)+\frac{\omega}{\omega_{s}} i_{q 1}+\frac{R_{s 1}}{L_{s 1}} i_{d 1}-\frac{V_{1}}{L_{s 1}} \sin \theta_{1}(7) \\
& \frac{1}{\omega_{s}} \frac{d}{d t} i_{d 2}=\frac{R_{s 2}}{L_{s 2}} i_{d 2}+\frac{\omega}{\omega_{s}} i_{q 2}-\frac{K_{2}}{L_{s 2}} \cos \left(\alpha_{2}-\theta_{1}\right) V d c- \\
& \frac{1}{L_{s 2}}\left(V_{2} \sin \theta_{2}-V_{1} \sin \theta_{1}\right) \\
& \frac{1}{\omega_{s}} \frac{d}{d t} i_{q 2}=\frac{R_{s 2}}{L_{s 2}} i_{d 2}+\frac{\omega}{\omega_{s}} i_{q 2}+\frac{K_{2}}{L_{s 2}} \sin \left(\alpha_{2}-\theta_{1}\right) V d c- \\
& \frac{1}{L_{s 2}}\left(V_{2} \sin \theta_{2}-V_{1} \sin \theta_{1}\right) \\
& \frac{c}{\omega_{s}} \frac{d}{d t} V_{d c}=-k_{1} \cos \left(\alpha_{1}+\theta_{1}\right) i_{d 1}-k_{1} \sin \left(\alpha_{1}+\theta_{1}\right) i_{q 1}- \\
& k_{2} \cos \left(\alpha_{1}+\theta_{1}\right) i_{d 2}-k_{2} \sin \left(\alpha_{1}+\theta_{1}\right) i_{q 2}
\end{aligned}
$$

$$
\begin{aligned}
& V_{1} \sum_{j=1}^{n} V_{j} Y_{1 j} \cos \left(\theta_{1}-\theta_{j}-\emptyset_{1 j}\right) \\
& \left.0=V_{1}\left(i_{d 1}-i_{d 2}\right) \sin \theta_{1}+\left(i_{q 1}-i_{q 2}\right) \cos \theta_{1}\right)- \\
& V_{1} \sum_{j=1}^{n} V_{j} Y_{1 j} \sin \left(\theta_{1}-\theta_{j}-\emptyset_{1 j}\right) \\
& \text { Power at bus } 2 \text { (Kuwait) }
\end{aligned}
$$

$0=V_{2}\left(i_{d 2} \cos -i_{d 2} \sin \theta_{2}\right)-V_{2} \sum_{j=1}^{n} V_{j} Y_{2 j} \cos \left(\theta_{2}-\theta_{j}-\emptyset_{2 j}\right)$

$0=V_{2}\left(i_{d 2} \sin -i_{d 2} \cos \theta_{2}\right)-V_{2} \sum_{j=1}^{n} V_{j} Y_{2 j} \sin \left(\theta_{2}-\theta_{j}-\emptyset_{2 j}\right)$

Where $i_{d}$ and $i_{q}$ are injected dq of STATCOM current, $V_{d c}$ is the voltage across the dc capacitor, $\mathrm{R}_{\mathrm{dc}}$ represents the switching losses, $\mathrm{R}_{\mathrm{s}}$ and $\mathrm{L}_{\mathrm{s}}$ are coupling transformer resistance and inductance respectively, the Electrical power balance equation at bus 2 where STATCOM is implemented [5]. $V_{1} \angle \theta_{1}$ and $V_{2} \angle \theta_{2}$ are the terminal voltage of the SSSC and power balanced equation as demonstrated above at bus 1 is given above.

\section{Acknowledgement}

The authors greatly acknowledge the Late (Prof. Dr. Abdel Aty Edris) for his outstanding contribution to perform EMTP and TNA analyses during study and my Princesses Fatima Masood kept me busy full time did not allow me to carry-out my research.

\section{References}

[1] Therese Okeke and Ramy Zaher Flexible AC Transmission Systems (FACTS) published IEEE Conference, 2013

[2] Michael Bocovich and Ned Mohan "Overview of Series Connected Flexible AC Transmission Systems (FACTS)" published IEEE Conference, 2013

[3] X.Y Zhou and R.K Aggarwal "Detailed modelling and simulation of UPFC using EMTP" published IEEE Conference, 2014

[4] Muasam Yadav and Anukar Soni "Improvement of power flow and voltage stability using unified power flow controller" published in ICEEOT 2016.

[5] Tariq Masood, S.A. Qureshi "FACTS Control Devices (STATCOM, SSSC and UPFC) Re-Configuration Techniques By PSIM/MATLAB" IEEE-ICEE 9-12 April,2017, Lahore Pakistan 